\title{
Albumin and its Perspectives in Urolithiasis
}

\author{
Devesh Raizada, Pragya Kumar, Tanya Singh, Trisha Pruthi, Priyadarshini * \\ Jaypee Institute of Information Technology, A-10, Sector-62, Noida, Uttar Pradesh, India
}

Address for Correspondance: Priyadarshini, priyadarshini@jiit.ac.in ; tstanya650@gmail.com ; deveshraizada2495@gmail.com ; trishapruthi1@gmail.com; prags955@hotmail.com

\section{Keywords}

Urolithiasis;

Calculus; Albumin;

Therapeutic Agent;

Organic Matter.

\begin{abstract}
Urolithiasis is referred to as the formation of calculus in the kidneys. It is a disease with an incidence rate of more than one million cases being reported annually in India. There are various forms of the calculus and all of them have been reported to have $90-95 \%$ inorganic and $5 \%$ organic matter. Out of the major proteins that comprise of this organic component of the matrix, Albumin and Uromodulin are found to be the most abundant. Albumin is also the most abundant protein in the human blood serum where it plays the role of a transporter of hormones, fatty acids and other compounds. It is an antioxidant and is also known for maintaining $\mathrm{pH}$ and oncotic pressure. The prominence of this protein in urine as well as in the renal calculi brings to question whether or not it plays an important role in nephrolithiasis. Under several diseased conditions the concentration of albumin can be abnormally high in the urine, it may be possible that the increased concentrations of albumin may significantly affect a patient's susceptibility to kidney stone formation. Therefore, the incidence of certain diseases may indirectly end up affecting urolithiasis. Albumin, as a therapeutic agent has been used in restoring blood volumes in the case of traumas, burns and surgeries; it has also been used in the treatment of cirrhosis in an attempt to improve renal functions and circulation. The study of the role of albumin in urolithiasis could give us useful insights on its potential role in this disease and may add to the therapeutic repertoire of albumin. (C) 2016 iGlobal Research and Publishing Foundation. All rights reserved.
\end{abstract}

Conference Proceedings: International Conference on Advances in Plant and Microbial Biotechnology (PMB-2017); JIIT, Noida: February 02-04, 2017

Indo Global Journal of Pharmaceutical Sciences( ISSN 22491023 ; CODEN- IGJPAI; NLM ID: 101610675) indexed and abstracted in EMBASE(Elsevier), SCIRUS(Elsevier),CABI, CAB Abstracts, Chemical Abstract Services(CAS), American Chemical Society(ACS), Index Copernicus, EBSCO, DOAJ, Google Scholar and many more. For further details, visit http://iglobaljournal.com 